\title{
Decomposing a Scene into Geometric and Semantically Consistent Regions
}

\author{
Stephen Gould \\ Dept. of Electrical Engineering \\ Stanford University \\ sgouldestanford.edu
}

\author{
Richard Fulton \\ Dept. of Computer Science \\ Stanford University \\ rafultonecs.stanford.edu
}

\author{
Daphne Koller \\ Dept. of Computer Science \\ Stanford University \\ kollerecs.stanford.edu
}

\begin{abstract}
High-level, or holistic, scene understanding involves reasoning about objects, regions, and the $3 D$ relationships between them. This requires a representation above the level of pixels that can be endowed with high-level attributes such as class of object/region, its orientation, and (rough 3D) location within the scene. Towards this goal, we propose a region-based model which combines appearance and scene geometry to automatically decompose a scene into semantically meaningful regions. Our model is defined in terms of a unified energy function over scene appearance and structure. We show how this energy function can be learned from data and present an efficient inference technique that makes use of multiple over-segmentations of the image to propose moves in the energy-space. We show, experimentally, that our method achieves state-of-the-art performance on the tasks of both multi-class image segmentation and geometric reasoning. Finally, by understanding region classes and geometry, we show how our model can be used as the basis for $3 D$ reconstruction of the scene.
\end{abstract}

\section{Introduction}

With recent success on many vision subtasks - object detection $[21,18,3]$, multi-class image segmentation $[17,7$, $13]$, and $3 \mathrm{D}$ reconstruction $[10,16]$-holistic scene understanding has emerged as one of the next great challenges for computer vision $[11,9,19]$. Here the aim is to reason jointly about objects, regions and geometry of a scene with the hope of avoiding the many errors induced by modeling these tasks in isolation.

An important step towards the goal of holistic scene understanding is to decompose the scene into regions that are semantically labeled and placed relative to each other within a coherent scene geometry. Such an analysis gives a highlevel understanding of the overall structure of the scene, allowing us to derive a notion of relative object scale, height above ground, and placement relative to important semantic categories such as road, grass, water, buildings or sky. We provide a novel method that addresses this goal.
Our method is based on a unified model where each pixel in the image is assigned to a single region. Regions are labeled both with a semantic category (such as grass, sky, foreground, and so on) and a geometric label (currently vertical, horizontal, or sky). Unlike methods that deal only with multi-class segmentation [17] or only with geometric reconstruction [10], our approach reasons jointly about both aspects of the scene, allowing us to avoid inconsistencies (such as vertical roads) and to utilize the context to reduce false positives (such as unsupported objects).

A key aspect of our approach is the use of large, dynamically-defined regions as the basic semantic unit. Most previous methods for doing this type of image decomposition use either individual pixels [17] or predefined superpixels [24, 5]. Each of these approaches has its tradeoffs. The use of individual pixels makes it difficult to utilize more global cues, including both robust statistics about the appearance of larger regions, which can help average out the random variations of individual pixels, and relationships between regions, which are hard to "transmit" by using local interactions at the pixel level. The use of superpixels partially addresses some of these concerns, but as superpixels are constructed in advance using simple procedures based on local appearance alone, their boundaries are often inconsistent with the true segment boundaries, making an accurate decomposition of the image impossible. Our approach dynamically associates pixels to regions, allowing region boundaries to adjust so as to accurately capture the true object boundaries. Moreover, our regions are also much larger than superpixels, allowing us to derive global appearance properties for each region, including not only color and texture, but even larger properties such as its general shape, aspect ratio, and characteristics of its boundary. These features can help capture subtle yet important cues about regions that improve classification accuracy. As we will see, this provides a decomposition of the scenes into objects or appearance-coherent parts of objects (such as person's head, or a window in a building).

Reasoning in our model requires that we infer both the pixel-to-region association and the semantic and geometric 
labels for the regions. We address this challenge using a hybrid approach. For the pixel-association task, we propose a novel multiple-segmentation approach, in which different precomputed segmentations are used to propose changes to the pixel-region associations. These proposed moves take large steps in the space and hence help avoid local minima; however, they are evaluated relative to our global energy function, ensuring that each step improves the energy. The region-labeling task is addressed using global energyminimization methods over the region space. This step is not too expensive, since the number of regions is significantly lower than the number of pixels. By performing the inference at this level, we also improve labeling accuracy because the adjacency structure between these larger regions allows us to directly exploit correlations between them (such as the fact that ground is below sky).

The parameters of our model are entirely learned from data. In this model, we are learning to label entire segments, allowing us to exploit global, region-level characteristics. We obtain positive examples for region labels from a large training set, which we constructed using Amazon Mechanical Turk (AMT), at a total cost of less than $\$ 250$. Negative examples are a bit trickier to acquire, as there are exponentially many "non-regions," most of which are obviously bad choices. We therefore propose a novel closed-loop training regime, where the algorithm runs inference on the training images given its current model, and then uses mistakes made in the process as negative examples to retrain.

We apply our method to a challenging data set consisting of 715 images, most of which have fairly low resolution and multiple small objects at varying scales. We show that our approach produces multi-class segmentation and surface orientation results that outperform state-of-the-art methods. In addition, we show how our output can be used as the basis for 3D scene reconstruction.

\section{Background and Related Work}

Our work touches on many facets of computer vision that have, in recent years, been treated as separate problems. The problem of multi-class image segmentation (or labeling) has been successfully addressed by a number of works $[7,22,17,23,24,5]$. The goal here is to label every pixel in the image with a single class label. Typically these algorithms construct CRFs over the pixels (or small coherent regions called superpixels) with local class-predictors based on pixel appearance and a pairwise smoothness term to encourage neighboring pixels to take the same label. Some novel works introduce 2D layout consistency between objects [23], object shape [22], or relative location between regions $[7,5]$. However, none of these works take into account 3D context and do not learn or enforce global consistency, such as that "sky" needs to be above "ground".

As an alternative to segmenting into semantic classes,
Hoiem et al. [12] propose segmenting free-standing objects by estimating occlusion boundaries in an image. Other works attempt to reconstruct 3D depth [16] or surface geometry [10] directly from monocular images without first reasoning about occlusions. These use local color and texture cues together with pairwise interactions to infer scene structure. None of these works attempt to understand the semantic content of the scene and they tend to produce poor $3 \mathrm{D}$ reconstructions when foreground objects are present.

The use of multiple over-segmented images is not new to computer vision. Russell et al. [14], for example, use multiple over-segmentations for finding objects in images, and many of the depth reconstruction methods described above (e.g., [10]) make use of over-segmentations for computing feature statistics. In the context of multi-class image segmentation, Kohli et al. [13] specify a global objective which rewards solutions in which an entire segment is labeled consistently. However, their energy function is very restricted and does not, for example, capture the interaction between region appearance and class label nor does their energy function allow for label-dependent pairwise preferences, such as foreground objects above road. Unlike all of these methods, our method uses multiple oversegmentations to build a dictionary of proposal moves for optimizing a global energy function-the segments themselves are not used for computing features nor do they appear explicitly in our objective.

The importance of holistic scene interpretation has been highlighted in a number of recent works [11,9]. These methods combine tasks by passing the output of one model to the input of another. Unlike these approaches, which optimize variables for each task separately, our method considers semantic and geometric tasks simultaneously and performs joint optimization on a unified objective over the variables, providing a coherent decomposition of the scene.

Perhaps most relevant is the work of Tu et al. [20], which decomposes a scene into regions, text and faces using an innovative data driven MCMC approach on a generative model of the scene. However, their work is primarily focussed on identifying text and faces, and does not attempt to label "generic" regions with semantic classes, nor do they model the geometric relationship between regions.

\section{Region-based Scene Decomposition}

Our goal is to decompose an image $\mathcal{I}$ into an unknown number $(K)$ of geometrically and semantically consistent regions by iteratively optimizing an energy function that measures the quality of the solution at hand. We begin by describing the various entities in our model. Inference and learning are described in Section 4.

Our model reasons about both pixels and regions. Each pixel in the image $p \in \mathcal{I}$ belongs to exactly one region, which is identified by the pixel's region-correspondence 
variable $R_{p} \in\{1, \ldots, K\}$. Let the set of pixels in region $r$ be denoted by $\mathcal{P}_{r}=\left\{p: R_{p}=r\right\}$. The size of the region (number of pixels) is then $N_{r}=\left|\mathcal{P}_{r}\right|=\sum_{p} \mathbf{1}\left\{R_{p}=r\right\}$. Each pixel has a local appearance feature vector $\alpha_{p} \in \mathbb{R}^{n}$ (described in Section 3.1 below). Associated with each region are: a semantic class label $S_{r}$, currently grass, mountain, water, sky, road, tree, building and foreground; a geometry $G_{r}$, currently horizontal, vertical, and sky; and an appearance $A_{r}$ that summarizes the appearance of the region as a whole. The final component in our model is the horizon. We assume that the image was taken by a camera with horizontal axis parallel to the ground. We therefore model the location of the horizon as the row in the image corresponding to the horizon $v^{\text {hz }} \in\{1, \ldots$, height $(\mathcal{I})\}$.

Given an image $\mathcal{I}$ and model parameters $\boldsymbol{\theta}$, our unified energy function scores the entire description of the scene: the pixel-to-region associations $\mathbf{R}$; the region semantic class labels $\mathbf{S}$, geometries $\mathbf{G}$, and appearances $\mathbf{A}$; and the location of the horizon $v^{\mathrm{hz}}$ :

$$
\begin{aligned}
& E \\
& \quad\left(\mathbf{R}, \mathbf{S}, \mathbf{G}, \mathbf{A}, v^{\text {hz }}, K \mid \mathcal{I}, \boldsymbol{\theta}\right)= \\
& \quad+\theta^{\text {horizon }} \psi^{\text {horizon }}\left(v^{\mathrm{hz}}\right) \\
& \quad+\theta^{\text {region }} \sum_{r} \psi_{r}^{\text {region }}\left(S_{r}, G_{r}, v^{\mathrm{hz}} ; A_{r}, \mathcal{P}_{r}\right) \\
& \quad+\theta^{\text {pair }} \sum_{r s} \psi_{r s}^{\text {pair }}\left(S_{r}, G_{r}, S_{s}, G_{s} ; A_{r}, \mathcal{P}_{r}, A_{s}, \mathcal{P}_{s}\right) \\
& \quad+\theta^{\text {boundary }} \sum_{p q} \psi_{p q}^{\text {boundary }}\left(R_{p}, R_{q} ; \alpha_{p}, \alpha_{q}\right) .
\end{aligned}
$$

We now describe each of the components of our model.

\subsection{Characterizing Individual Region Appearance}

For each pixel $p$ in the image, we construct a local appearance descriptor vector $\alpha_{p}$ comprised of raw image features and discriminitively learned boosted features. Our raw image features, which are computed in a small neighborhood of the pixel, are identical to the 17-dimensional color and texture features described in [17]. We augment these raw features with more processed summaries that represent the "match" between the pixel's local neighborhood and each of the region labels. In particular, for each (individual) semantic and geometric label we learn a one-vs-all boosted classifier to predict the label given the raw image features in a small neighborhood around the pixel. ${ }^{1}$ We then append the score (log-odds ratio) from each boosted classifier to our pixel appearance feature vector $\alpha_{p}$.

In our experiments, we set the region appearance $A_{r}$ to be the maximum-likelihood Gaussian parameters over the appearance of pixels within the region: $A_{r}=\left(\mu_{r}^{A}, \Sigma_{r}^{A}\right)$ where $\mu_{r}^{A} \in \mathbb{R}^{n}$ and $\Sigma_{r}^{A} \in \mathbb{R}^{n \times n}$ are the mean and covariance matrix for the appearance vectors $\alpha_{p}$ of pixels in

\footnotetext{
${ }^{1}$ In our experiments we append to the pixel's 17 features, the average and variance for each feature over a $5 \times 5$-pixel window in 9 grid locations around the pixel and the image row to give a total of 324 features. We use the GentleBoost algorithm with 2-split decision stumps and train for 500 rounds. Our results appeared robust to the choice of parameters.
}

the $r$-th region. These summary statistics give us a more robust estimator of the appearance of the region than would be obtained by considering only small neighborhoods of the individual pixels.

\subsection{Individual Region Potentials}

To define the potentials that help infer the label of individual regions, we extract features $\phi_{r}\left(A_{r}, \mathcal{P}_{r}\right) \in \mathbb{R}^{n}$ describing the region appearance and basic shape. Our appearance features include the mean and covariance $\mu_{r}^{A}, \Sigma_{r}^{A}$, the log-determinant of $\Sigma_{r}^{A}$, and the average contrast at the region boundary and region interior. In addition to relating to semantic class-grass is green-the appearance features provide a measure for the quality of a region-well-formed regions will tend to have strong boundary contrast and (depending on the class) little variation of interior appearance.

We also want to capture more global characteristics of our larger regions. For example, we would like to capture the fact that buildings tend to be vertical with many straight lines, trees tend to be green and textured, and grass tends to be green and horizontal. Thus, we incorporate shape features that include normalized region area, perimeter, first and second $x$ - and $y$-moments, and residual to a robust line fit along the top and bottom boundary of the region. The latter features capture the fact that buildings tend to have straight boundaries while trees tend to be rough.

We also include the horizon variable in the regionspecific potential, allowing us to include features that measure the ratio of pixels in the region above and below the horizon. These features give us a sense of the scale of the object and its global position in the scene. For example, buildings are tall and tend to have more mass above the horizon than below it; foreground objects are often close and will have most of their mass below the horizon. Conversely, these potentials also allow us to capture the strong positional correlation between the horizon and semantic classes such as sky or ground, allowing us to use the same potential to place the horizon within the image.

To put all of these features together, we learn a multiclass logistic classifier for $S_{r} \times G_{r}$ with a quadratic kernel over $\phi_{r}$ (see Section 4.2). The score for any assignment to the region variables is then: $\psi_{r}^{\text {region }}\left(S_{r}, G_{r}, v^{\text {hz }} ; A_{r}, \mathcal{P}_{r}\right)=$ $-N_{r} \log \sigma\left(S_{r} \times G_{r} \mid \phi_{r}\left(A_{r}, \mathcal{P}_{r}\right), v^{\mathrm{hz}}\right)$, where $\sigma(\cdot)$ is the multi-class logistic function with learned parameters. We scale the potential by the region size $N_{r}$ so that our score gives more weight to larger regions and is independent of the number of regions in the image.

\subsection{Inter-Region Potentials}

Our model contains two types of inter-region potentials. The first of these is $\psi_{p q}^{\text {boundary }}\left(R_{p}, R_{q} ; \alpha_{p}, \alpha_{q}\right)$, which is the standard contrast-dependent pairwise boundary potential [17]. For two adjacent pixels $p$ and $q$, we de- 
fine $\psi_{p q}^{\text {boundary }}\left(R_{p}, R_{q} ; \alpha_{p}, \alpha_{q}\right)=\exp \left\{-\beta^{-1}\left\|\alpha_{p}-\alpha_{q}\right\|^{2}\right\}$ if $R_{p} \neq R_{q}$ and zero otherwise where $\beta$ is half the average contrast between all adjacent pixels in the image. This term penalizes adjacent regions that do not have an edge between them; it has the effect of trying to merge adjacent regions that are not clearly demarcated. We note that, since the penalty is accumulated over pairs of adjacent pixels, the region-level penalty is proportional to the pixel-length of the boundary between the regions.

Our second inter-region potential, $\psi^{\text {pair }}$, models the affinity of two classes to appear adjacent to each other. Similar to the within-region potentials, we extract features $\phi_{r s}\left(A_{r}, \mathcal{P}_{r}, A_{s}, \mathcal{P}_{s}\right) \in \mathbb{R}^{m}$ for every pair of adjacent regions $r$ and $s$. We then learn independent multi-class logistic classifiers for $S_{r} \times S_{s}$ and $G_{r} \times G_{s}$ given these features. Note that these potentials are asymmetric (exchanging regions $r$ and $s$ gives a different preference). The features $\phi_{r s}$ are intended to model contextual properties between regions, for example, the boundary between building and sky tends to be straight and building is more likely to appear above a foreground object than below it. To capture these properties, our features include the difference between centroids of the two regions, the proportion of pixels along the boundary in which region $r$ is above region $s$, the length and orientation of the boundary, and residual in fitting a straight line to the boundary. In addition to these layout-based features, we include appearance difference between the regions normalized by the total appearance variance within each region. This captures signals such as foreground objects tend to contrast highly with other regions, whereas background regions are more similar in appearance, such as adjacent buildings in a city.

We normalize each pairwise potential by the sum of the number of pixels in each region divided by the number of neighbors for the region: $\eta=\left(\frac{N_{r}}{|\operatorname{nbrs}(r)|}+\frac{N_{s}}{|\operatorname{nbrs}(s)|}\right)$. This makes the total influence on a region independent of its number of neighbors while still giving larger regions more weight. The final form of our second inter-region potential is then $\psi_{r s}^{\text {pair }}\left(S_{r}, G_{r}, S_{s}, G_{s} ; A_{r}, \mathcal{P}_{r}, A_{s}, \mathcal{P}_{s}\right)=$ $-\eta \log \sigma\left(S_{r} \times S_{s} \mid \phi_{r s}\right)-\eta \log \sigma\left(G_{r} \times G_{s} \mid \phi_{r s}\right)$, where, as above, $\sigma(\cdot)$ is the multi-class logistic function.

\section{Inference and Learning}

\subsection{Inference Algorithm}

Exact inference in our model is clearly intractable. We adopt a two-stage hill climbing approach to minimize the energy. In the first stage, we modify the pixel-region association variables by allowing a set of pixels to change the region to which they are assigned. Given the new pixel assignments, we then optimize the region and horizon variables in the second stage. The global energy of the resulting configuration is then evaluated, and the move is accepted

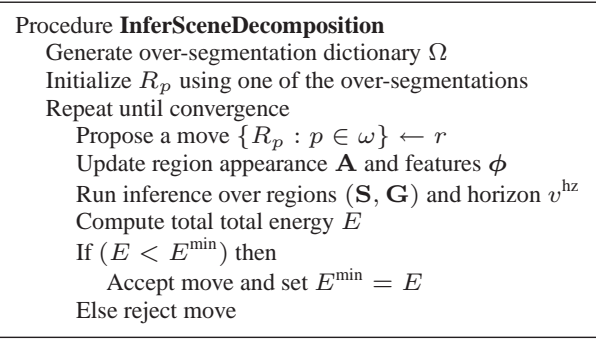

(a)

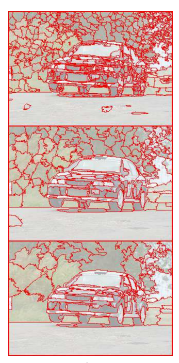

(b)
Figure 1. (a) Scene decomposition inference algorithm; (b) Oversegmentation dictionary, $\Omega$, generated by running mean-shift [1] with three different parameter settings. See text for details.

only if this energy improves, ensuring that our inference is continuously improving a coherent global objective.

The proposal moves for region associations are drawn from a pre-computed, image-specific dictionary of image segments $\Omega$ (Figure 1(b)). To build a "good" set of segments we start with a number of different over-segmentations of the image. Here, we use the mean-shift algorithm [1] using publicly available code. ${ }^{2}$ We generate different oversegmentations by varying the spatial and range bandwidth parameters. To allow coarse granularity moves, we also perform hierarchical agglomerative clustering (up to a fixed depth) on each over-segmentation by merging adjacent segments that have similar appearance. We then add all subsets constructed by this process (including the initial segments) to the dictionary. This procedure produces a rich set of proposal moves. We sort the dictionary by the entropy of pixel appearance within each segment so that more uniform segments are proposed first.

In addition to moves proposed by the dictionary, we also allow moves in which two adjacent regions are merged together. The set of allowed pixel-to-region correspondence proposal moves is thus: (i) pick a segment $\omega \in \Omega$ and assign all $R_{p}$ for $p \in \omega$ to a new region; (ii) pick a segment $\omega \in \Omega$ and assign all $R_{p}$ for $p \in \omega$ to one of the regions in its neighborhood; or (iii) pick two neighboring regions $r$ and $s$ and merge them, that is, $\forall R_{p}=s$ set $R_{p}=r$.

Our overall inference algorithm is summarized in Figure 1(a): Briefly, we initialize our pixel-to-region associations $\mathbf{R}$ using one of the over-segmentations used to produce our dictionary. Given our current association $\mathbf{R}$, we select a proposal move and reassign pixels to form new regions. We then update the appearance model $A_{r}$ and features of any region that was affected by the move. We maintain sufficient statistics over pixel appearance, making this step very fast. Keeping the pixel-to-region correspondence variables and horizon fixed, we run max-product belief propagation on the region class and geometry variables.

\footnotetext{
${ }^{2}$ http://www. caip.rutgers.edu/riul/research/ code/EDISON/index.html
} 
We then update the horizon $v^{\mathrm{hz}}$ using Iterated Conditional Modes (ICM). ${ }^{3}$ The new configuration is evaluated relative to our global energy function, and kept if it provides an improvement. The algorithm iterates until convergence. In our experiments (Section 5) inference took between 30 seconds and 10 minutes to converge depending on image complexity (i.e., number of segments in $\Omega$ ).

\subsection{Learning Algorithm}

We train our model using a labeled dataset where each image is segmented into regions that are semantically and geometrically coherent. Thus, our ground truth specifies both the region association for each pixel and the labels for each region.

We learn each term $\psi^{\text {horizon }}, \psi^{\text {region }}$ and $\psi^{\text {pair }}$ in our energy function separately, using our labeled training data. We then cross-validate the weights between the terms using a subset of our training data. Since only the relative weighting between terms matter, we fixed $\theta^{\text {region }}$ to one.

For the horizon singleton term, we learn a Gaussian over the location of the horizon in training images and set $\psi^{\text {horizon }}\left(v^{\mathrm{hz}}\right)$ to be the log-likelihood of $v^{\mathrm{hz}}$ given this model. We normalize $\psi^{\text {horizon }}\left(v^{\mathrm{hz}}\right)$ by the image height to make this model resolution invariant. Our learned Gaussian has a mean of approximately 0.5 and standard deviation of 0.15 (varying slightly across experiment folds). This suggests that the horizon in our dataset is quite well spread around the center of the image.

The within-region term, $\psi^{\text {region }}$, and the between-region term, $\psi^{\text {pair }}$, are learned using multi-class logistic regression. However, the training of the within-region term involves an important subtlety. One of the main roles of this term is to help recognize when a given collection of pixels is actually a coherent region-one corresponding to a single semantic class and a single geometry. Although all of the regions in our training set are coherent, many of the moves proposed during the course of inference are not. For example, our algorithm may propose a move that merges together pixels containing (horizontal) grass and pixels containing (vertical) trees. We want to train our classifier to recognize invalid moves and penalize them. To penalize such moves, we train our multi-class logistic regression classifier with an additional "invalid" label. This label cannot be assigned to a candidate region during inference, and so if the proposed region $r$ appears incoherent, the "invalid" label will get high probability, reducing the probability for all (valid) labels in $S_{r} \times G_{r}$. This induces a high energy for the new proposed assignment, making it likely to be rejected.

To train a discriminative classifier that distinguishes be-

\footnotetext{
${ }^{3}$ We experimented with including $v^{\mathrm{hz}}$ in the belief propagation inference but found that it changed very little from one iteration to the next and was therefore more efficient to infer conditionally (using ICM) once the other variables were assigned.
}

tween coherent and incoherent regions, we need to provide it with negative (incoherent) training instances. Here, we cannot simply collect arbitrary subsets of adjacent pixels that do not correspond to coherent regions: Most arbitrary subsets of pixels are easily seen to be incoherent, so that a discriminative model trained with such subsets as negative examples is unlikely to learn a meaningful decision boundary. Therefore, we use a novel "closed-loop" learning procedure, where the algorithm trains on its own mistakes. We begin by training our classifier where the negative examples are defined by merging pairs of adjacent ground truth regions (which are not consistent with each other). We then perform inference (on our training set) using this model. During each proposal move we evaluate the outcome of inference with the ground truth annotations. We append to our training set moves that were incorrectly accepted or rejected, or moves that were accepted (resulted in lower energy) but produced an incorrect labeling of the region variables. In this way, we can target the training of our decision boundary on the more troublesome examples.

\section{Experimental Results}

We conduct experiments on a set of 715 images of urban and rural scenes assembled from a collection of public image datasets: LabelMe [15], MSRC [2], PASCAL [4], and Geometric Context (GC) [10]. Our selection criteria were for the images to have approximately $320 \times 240$ pixels, contain at least one foreground object and have the horizon positioned within the image (it need not be visible). We perform 5-fold cross-validation with the dataset randomly split into 572 training images and 143 test images for each fold. The quality of our annotations (obtained from Amazon Mechanical Turk) is extremely good and in many cases superior to those provided by the original datasets. Images and labels are available for download from the first author's website.

Baselines. To validate our method and provide strong baselines for comparison, we performed experiments on independent multi-class image segmentation and geometry prediction using standard pixelwise CRF models. Here the pixel class $S_{p}$ (or surface geometry $G_{p}$ ) is predicted separately for each pixel $p \in \mathcal{I}$ given the pixel's appearance $\alpha_{p}$ (see Section 3.1). A contrast-dependent pairwise smoothness term is added to encourage adjacent pixels to take the same value. The models have the form

$$
E(\mathbf{S} \mid \mathcal{I})=\sum_{p} \psi_{p}\left(S_{p} ; \alpha_{p}\right)+\theta \sum_{p q} \psi_{p q}\left(S_{p}, S_{q} ; \alpha_{p}, \alpha_{q}\right)
$$

and similarly for $E(\mathbf{G} \mid \mathcal{I})$. In this model, each pixel can be thought of as belonging to its own region. The parameters are learned as described above with $\psi_{p}$ a multi-class logistic over boosted appearance features and $\psi_{p q}$ the boundary penalty. The baseline results are shown in Table 1. 


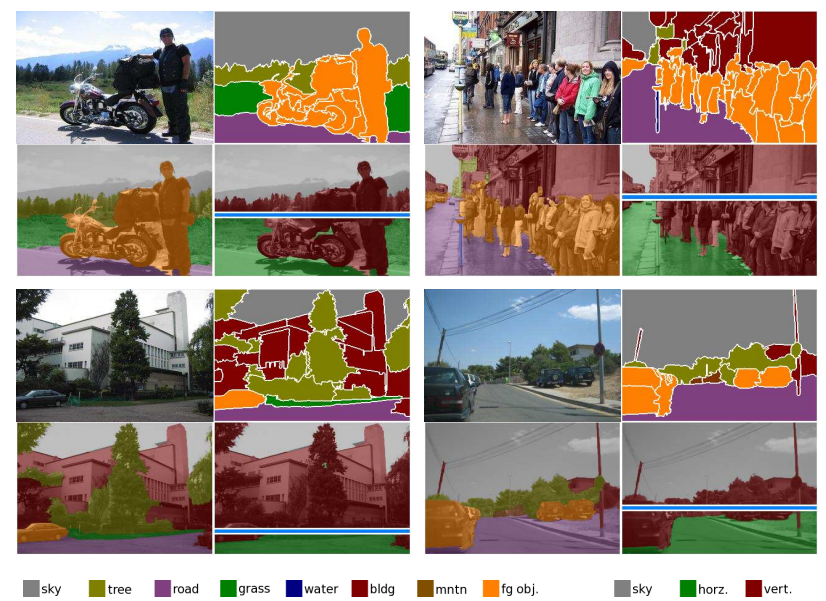

Figure 2. Examples of typical scene decompositions produced by our method. Show for each image are regions (top right), semantic class overlay (bottom left), and surface geometry with horizon (bottom right). Best viewed in color.

Region-based Approach. Multi-class image segmentation and surface orientation results from our region-based approach are shown below the baseline results in Table 1 . Our improvement of $2.1 \%$ over baseline for multi-class segmentation and $1.9 \%$ for surface orientation is significant. In particular, we observed an improvement in each of our five folds. Our horizon prediction was within an average of $6.9 \%$ (relative to image height) of the true horizon.

In order to evaluate the quality of our decomposition, we computed the overlap score between our boundary predictions and our hand annotated boundaries. To make this metric robust we first dilate both the predicted and ground truth boundaries by five pixels. We then compute the overlap score by dividing the total number of overlapping pixels by half the total number of (dilated) boundary pixels (ground truth and predicted). A score of one indicates perfect overlap. We averaged 0.499 across the five folds indicating that on average we get about half of the semantic boundaries correct. For comparison, using the baseline class predictions gives a boundary overlap score of 0.454 .

The boundary score result reflects our algorithm's tendency to break regions into multiple segments. For example, it tends to leave windows separated from buildings and people's torsos separated from their legs (as can be seen in Figure 2). This is not surprising given the strong appearance difference between these different parts. We hope to extend our model in the future with object specific appearance and shape models so that we can avoid these types of errors.

Figures 3 and 4 show some good and bad examples, respectively. Notice the high quality of the class and geometry predictions particularly at the boundary between classes and how our algorithm deals well with both near and far objects. There are still many mistakes that we would like to

\begin{tabular}{|l||cc||cc|}
\hline \multicolumn{1}{|c||}{} & \multicolumn{2}{c||}{ CLASS } & \multicolumn{2}{c|}{ GEOMETRY } \\
\hline Pixel CRF (baseline) & 74.3 & $(0.80)$ & 89.1 & $(0.73)$ \\
Region-based energy & $\mathbf{7 6 . 4}$ & $(1.22)$ & $\mathbf{9 1 . 0}$ & $(0.56)$ \\
\hline
\end{tabular}

Table 1. Multi-class image segmentation and surface orientation (geometry) accuracy. Standard deviation shown in parentheses.

\begin{tabular}{|l|c||c|c|}
\hline \multicolumn{2}{|c||}{ MSRC } & \multicolumn{2}{c|}{ GC } \\
\hline TextonBoost [17] & 72.2 & Hoiem et al. [10]: & \\
Yang et al. [24] & 75.1 & $\bullet$ pixel model & 82.1 \\
Gould et al. [5] & $\mathbf{7 6 . 5}$ & $\bullet$ full model & $\mathbf{8 8 . 1}$ \\
\hline Pixel CRF & 75.3 & Pixel CRF & 86.5 \\
Region-based & 76.4 & Region-based & 86.9 \\
\hline
\end{tabular}

Table 2. Comparison with state-of-the-art MSRC and GC results against our restricted model. Table shows mean pixel accuracy.

address in future work. For example, our algorithm is often confused by strong shadows and reflections in water as can be seen in some of the examples in Figure 4. We hope that with stronger geometric reasoning we can avoid this problem. Also, without knowledge of foreground subclasses, our algorithm sometimes merges a person with a building or confuses boat masts with buildings.

Comparison with Other Methods. We also compared our method with state-of-the-art techniques on the 21-class MSRC [2] and 3-class GC [10] datasets. To make our results directly comparable with published works, we removed components from our model not available in the ground-truth labels for the respective datasets. That is, for MSRC we only use semantic class labels and for GC we only use (main) geometry labels. Neither model used horizon information. Despite these restrictions, our regionbased energy approach is still competitive with state-of-theart. Results are shown in Table 2.

\section{Application to 3D Reconstruction}

The output of our model can be used to generate novel $3 \mathrm{D}$ views of the scene. Our approach is very simple and obtains its power from our region-based decomposition rather than sophisticated features tuned for the task. Nevertheless, the results from our approach are surprisingly good compared to the state-of-the-art (see Figure 5 for some examples). Since our model does not provide true depth estimates our goal here is to produce planar geometric reconstructions of each region with accurate relative distances rather than absolute distance. Given an estimate of the distance between any two points in the scene, our 3D reconstruction can then be scaled to the appropriate size.

Our rules for reconstruction are simple. Briefly, we assume an ideal camera model with horizontal $(x)$ axis parallel to the ground. We fix the camera origin at $1.8 \mathrm{~m}$ above the ground (i.e., $y=1.8$ ). We then estimate the $y z$-rotation of the camera from the location of the horizon (assumed to be at depth $\infty)$ as $\theta=\tan ^{-1}\left(\frac{1}{f}\left(v^{\mathrm{hz}}-v_{0}\right)\right)$ where $v_{0}$ is half 


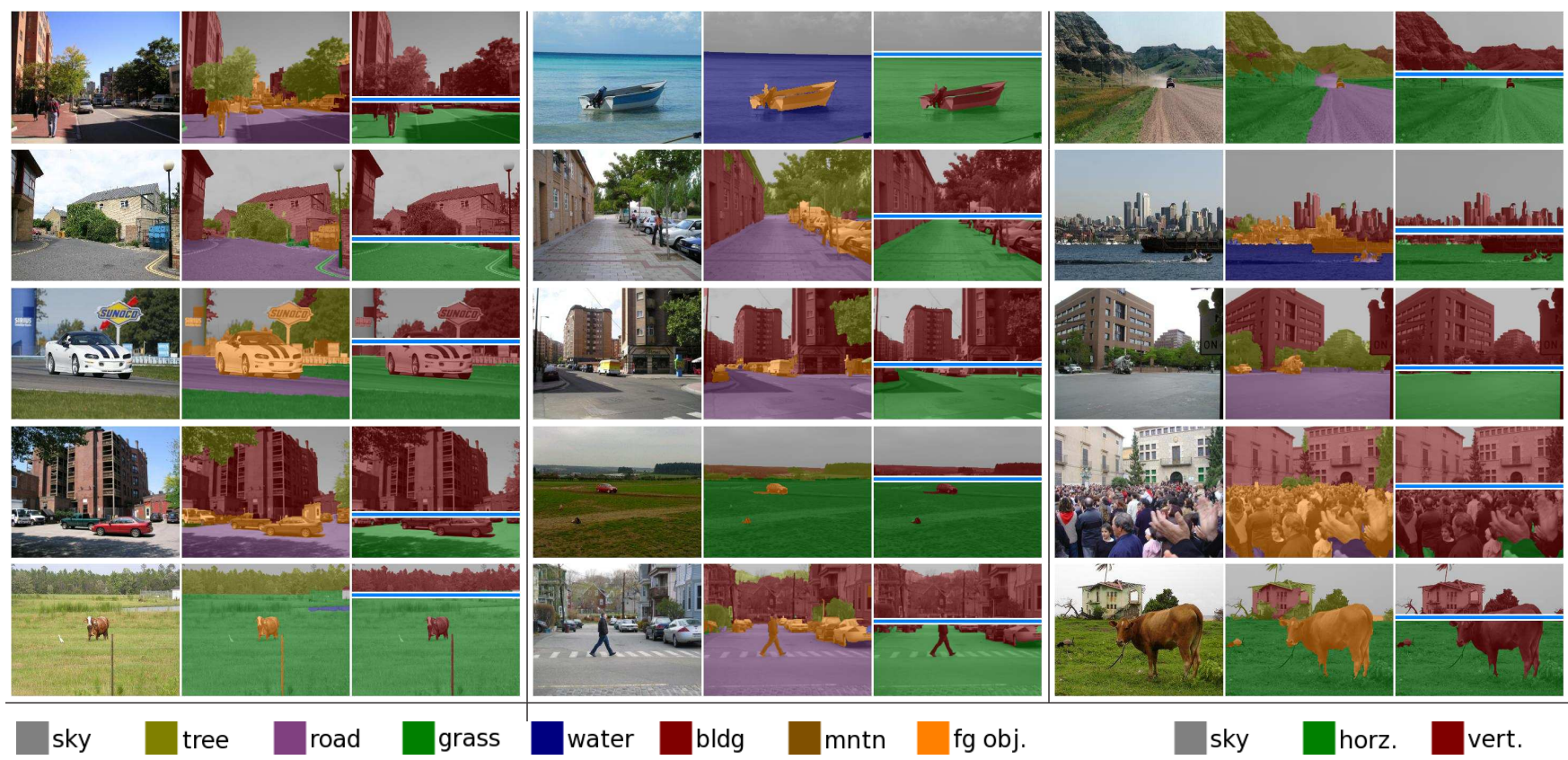

Figure 3. Representative results when our model does well. Each cell shows original image (left), overlay of semantic class label (center), and surface geometry (right) for each image. Predicted location of horizon is shown on the geometry image. Best viewed in color.
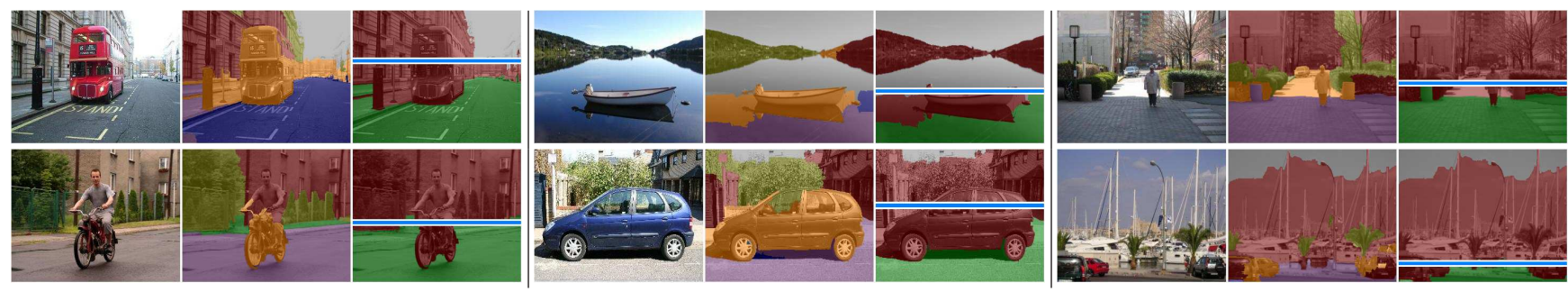

Figure 4. Examples of where our algorithm makes mistakes, such as mislabeling of road as water (top left), or confusing boat masts as buildings (bottom right). We also have difficulty with shadows and reflections. Best viewed in color.

the image height and $f$ is the focal length of the camera. Now the 3D location of every pixel $p=(u, v)$ lies along the ray $\mathbf{r}_{p}=\mathbf{R}(\theta)^{-1} \mathbf{K}^{-1}\left[\begin{array}{lll}u & v & 1\end{array}\right]^{T}$, where $\mathbf{R}(\theta)$ is the rotation matrix and $\mathbf{K}$ is the camera model [6]. It remains to scale this ray appropriately.

We process each region in the image depending on its semantic class. For ground plane regions (road, grass and water) we scale the ray to make the height zero. We model each vertical region (tree, building, mountain and foreground) as a planar surface whose orientation and depth with respect to the camera are estimated by fitting a robust line over the pixels along its boundary with the ground plane. This produced good results despite the fact that not all of these pixels are actually adjacent to the ground in 3D (such as the belly of the cow in Figure 5). When a region does not touch the ground (that is, it is occluded by another object), we estimate its orientation using pixels on its bottom-most boundary. We then place the region half way between the depth of the occluding object and maximum possible depth (either the horizon or the point at which the ground plane would become visible beyond the occluding object). The 3D location of each pixel $p$ in the region is determined by the intersection of this plane and the ray $\mathbf{r}_{p}$. Finally, sky regions are placed behind the last vertical region. ${ }^{4}$

\section{Discussion and Future Work}

In this work, we addressed the problem of decomposing a scene into geometrically and semantically coherent regions. Our method combines reasoning over both pixels and regions through a unified energy function. We proposed

\footnotetext{
${ }^{4}$ Technically, sky regions should be placed at the horizon, but since the horizon has infinite depth, we choose to render sky regions closer, so as to make them visible in our viewer.
} 

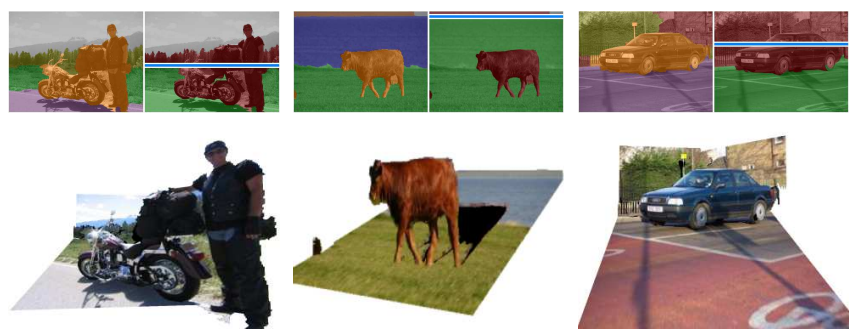

Figure 5. Novel views of a scene with foreground objects generated by geometric reconstruction.

an effective inference technique for optimizing this energy function and showed how it could be learned from data. Our results compete with state-of-the-art multi-class image segmentation and geometric reasoning techniques. In addition, we showed how a region-based approach can be applied to the task of 3D reconstruction, with promising results.

Our framework provides a basis on which many valuable extensions can be layered. With respect to 3D reconstruction, our method achieves surprising success given that it uses only simple geometric reasoning derived from the scene decomposition and location of the horizon. These results could undoubtedly be improved further by integrating our method with state-of-the-art approaches that reason more explicitly about depth [16] or occlusion [12].

An important and natural extension to our method can be provided by incorporating object-based reasoning directly into our model. Here, we can simply refine our foreground class into subclasses representing object categories (person, car, cow, boat, etc.). Such models would allow us to incorporate information regarding the relative location of different classes (cars are found on roads), which are very naturally expressed in a framework that explicitly models large regions and their (rough) relative location in 3D. By reasoning about different object classes, we can also incorporate state-of-the-art models regarding object shape [8] and appearance features [3]. We believe that this extension would allow us to address one of the important error modes of our algorithm, whereby foreground objects are often broken up into subregions that have different local appearance (a person's head, torso, and legs). Thus, this approach might allow us to decompose the foreground class into regions that correspond to semantically coherent objects (such as individual people or cars).

Finally, an important limitation of our current approach is its reliance on a large amount of hand-labeled training data. We hope to extend our framework to make use of large corpora of partially labeled data, or perhaps by using motion cues in videos to derive segmentation labels.

Acknowledgments. We give warm thanks to Geremy Heitz and Ben Packer for the many helpful discussions regarding this work. This work was supported by DARPA T/L SA4996-10929-4 and MURI contract N000140710747.

\section{References}

[1] D. Comaniciu, P. Meer, and S. Member. Mean shift: A robust approach toward feature space analysis. PAMI, 2002.

[2] A. Criminisi. Microsoft research cambridge object recognition image database. http://research.microsoft.com/vision/cambridge/recognition, 2004.

[3] N. Dalal and B. Triggs. Histograms of oriented gradients for human detection. In CVPR, 2005.

[4] M. Everingham, L. Van Gool, C. K. I. Williams, J. Winn, and A. Zisserman. The PASCAL Visual Object Classes Challenge 2007 (VOC2007) Results, 2007.

[5] S. Gould, J. Rodgers, D. Cohen, G. Elidan, and D. Koller. Multi-class segmentation with relative location prior. IJCV, 2008.

[6] R. I. Hartley and A. Zisserman. Multiple View Geometry in Computer Vision. Cambridge University Press, 2004.

[7] X. He, R. Zemel, and M. Carreira-Perpinan. Multiscale CRFs for image labeling. In CVPR, 2004.

[8] G. Heitz, G. Elidan, B. Packer, and D. Koller. Shape-based object localization for descriptive classification. In NIPS, 2008.

[9] G. Heitz, S. Gould, A. Saxena, and D. Koller. Cascaded classification models: Combining models for holistic scene understanding. In NIPS, 2008.

[10] D. Hoiem, A. A. Efros, and M. Hebert. Recovering surface layout from an image. IJCV, 2007.

[11] D. Hoiem, A. A. Efros, and M. Hebert. Closing the loop on scene interpretation. CVPR, 2008.

[12] D. Hoiem, A. N. Stein, A. A. Efros, and M. Hebert. Recovering occlusion boundaries. ICCV, 2007.

[13] P. Kohli, L. Ladicky, and P. Torr. Robust higher order potentials for enforcing label consistency. In CVPR, 08.

[14] B. C. Russell, A. A. Efros, J. Sivic, W. T. Freeman, and A. Zisserman. Using multiple segmentations to discover objects and their extent in image collections. In $C V P R, 06$.

[15] B. C. Russell, A. B. Torralba, K. P. Murphy, and W. T. Freeman. Labelme: A database and web-based tool for image annotation. IJCV, 2008.

[16] A. Saxena, M. Sun, and A. Y. Ng. Learning 3-D scene structure from a single still image. In PAMI, 2008.

[17] J. Shotton, J. Winn, C. Rother, and A. Criminisi. TextonBoost: Joint appearance, shape and context modeling for multi-class obj. rec. and seg. In ECCV, 2006.

[18] A. Torralba, K. P. Murphy, and W. T. Freeman. Contextual models for object detection using BRFs. In NIPS, 2005.

[19] Z. Tu. Auto-context and its application to high-level vision tasks. In $C V P R, 2008$.

[20] Z. Tu, X. Chen, A. L. Yuille, and S.-C. Zhu. Image parsing: Unifying segmentation, detection, and recognition. In ICCV, 2003.

[21] P. Viola and M. J. Jones. Robust real-time face detection. IJCV, 2004.

[22] J. Winn and N. Jojic. LOCUS: Learning object classes with unsupervised segmentation. In ICCV, 2005.

[23] J. Winn and J. Shotton. The layout consistent random field for recognizing and segmenting partially occluded objects. In $C V P R, 2006$.

[24] L. Yang, P. Meer, and D. J. Foran. Multiple class segmentation using a unified framework over mean-shift patches. In CVPR, 2007. 